\title{
Sobre inicios y cierres en el discurso político*
}

\author{
On startings and endings in the political discourse
}

\author{
Luis Cortés Rodríguez
}

Universidad de Almería, CySOC. Correo electrónico: lcortes@ual.es

\begin{abstract}
El análisis de exposiciones largas (por ejemplo, de diez o más minutos) exige la creación de unas unidades discursivas capaces de segmentar tales discursos más allá de las ya tradicionales unidades de procesamiento: los enunciados y sus subunidades los actos y los subactos. Este artículo pretende esbozar unas categorías superiores de división, así como su aplicación a los "Debates en torno al Estado de la Nación”, un debate político que se celebra anualmente en España. Hemos distinguido dos tipos de unidades mayores (unidades temático-textuales): por un lado, las secuencias y sus subunidades, el inicio, desarrollo y cierre, y, por otro lado, las subsecuencias, y sus subunidades el tema, subtema, sub(sub)tema, etc. En ellas, se integrarán las ya mencionadas unidades de procesamiento (unidades ilocutivo-tectuales). Tal segmentación permitirá dar una mayor uniformidad al estudio discursivo de los mecanismos mediante los cuales conseguimos efectos (cortesía, ironía, argumentación, racismo, etc.).
\end{abstract}

Palabras clave: discurso oral, unidades de segmentación, análisis del discurso político.

The analysis of long pieces of discourse (for example, those lasting ten or more minutes) requires the establishment of new discourse units in order to be able to segment such pieces beyond the traditional processing units, utterances and their subunits -acts and subacts. This article aims at outlining superior categories of division and their application to the "State of the Nation Debate", a political debate which takes place in Spain every year. Two different types of major units are distinguished (textual-theme units): on one hand, the sequences and its subunits -beginning, middle and end; and, on the other hand, the subsequences and its subunits -topic, sub-theme, sub-sub-theme, etc. In the latter, the already mentioned processing units (textual-illocutive units) will be integrated. Such segmentation will provide the study of the discursive devices by which we achieve effects -courtesy, irony, argument, racism, etc.- with a higher level of uniformity.

Key words: oral discourse, segmentation units, political discourse analysis.

En recuerdo de Ana María Vigara Tauste

Este trabajo forma parte de proyecto FFI2012-31699, "Los debates sobre el estado de la nación (1983-2011). Análisis pragmalingüístico”, concedido por el Ministerio de Economía y Competitividad de España. 


\section{INTRODUCCIÓN}

Cualquier intento de análisis de discursos orales más o menos extensos, como pueden ser intervenciones de diez o más minutos, se encuentra con un problema que no es baladí: la insuficiencia de las unidades ya tradicionales de segmentación del discurso oral, denominadas ya por nosotros como unidades de procesamiento (Cortés y Camacho 2005). Es verdad que en la tradición discursiva se ha hablado y se han considerado unidades mayores que estas, pero también es cierto el escaso interés y, por tanto, desarrollo que su estudio ha tenido en el análisis del discurso en general y en el oral en particular. Ello nos llevó a considerar (Cortés en prensa) para nuestros análisis, junto a las citadas unidades de procesamiento, cuya unidad esencial es el enunciado y las subunidades el acto discursivo y el subacto, otras unidades superiores, procesadas y pertenecientes al plano secuencial. las temático-textuales; por un lado, las secuencias como unidad principal y el inicio, desarrollo y cierre como subunidades, y, por otro, las subsecuencias como unidad principal y los temas, sub(sub)temas como subunidades (Cuadro 1).

\section{Cuadro 1. Unidades del discurso oral (Cortés, en prensa)}

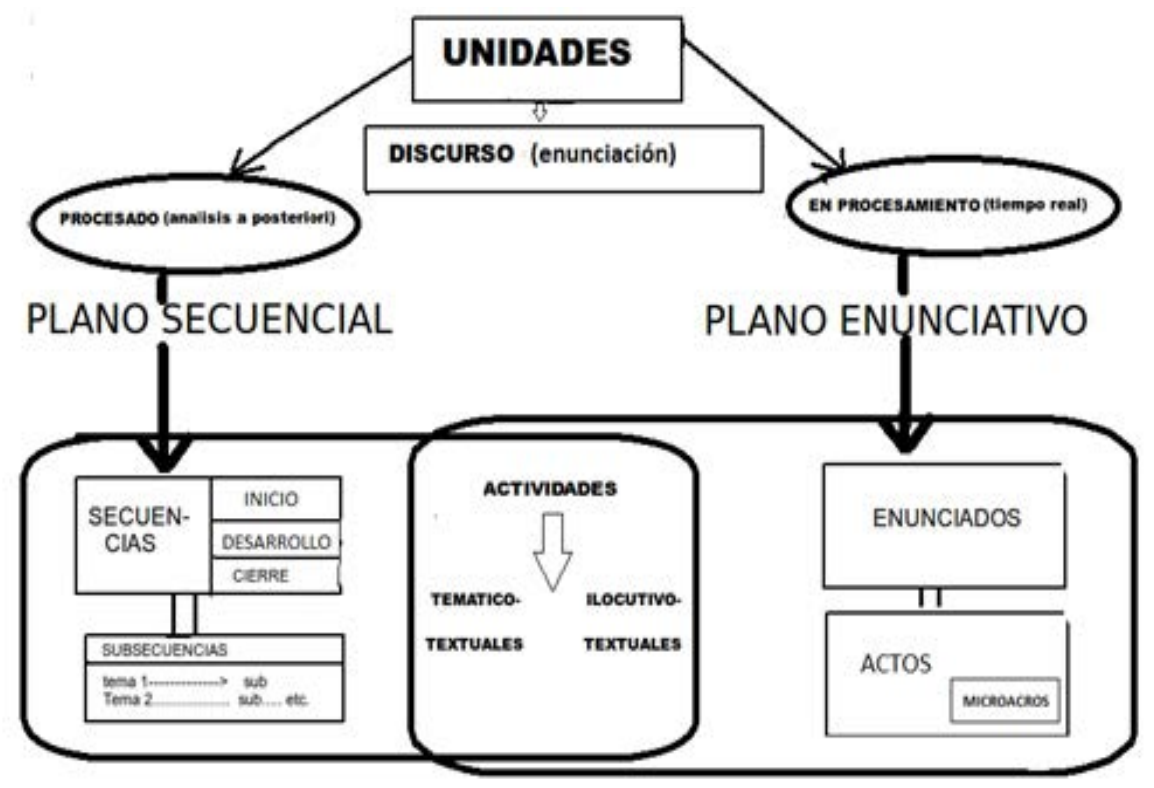

Como decíamos, han sido las de procesamiento las más estudiadas. En el mundo hispánico, hemos de destacar las aportaciones de los miembros del grupo de investigación Valesco (Universidad de Valencia). ${ }^{1}$ La bibliografía desarrollada ya es

\footnotetext{
Según ha reconocido recientemente su investigador principal (Briz 2011: 139) la propuesta del referido grupo se articula en tres dimensiones u órdenes, el estructural, el social y el informativo, y dos niveles, el monológico y el dialógico. Estructuralmente se organiza jerárquicamente en actos e intervenciones dentro del nivel monológico, y en intercambios y diálogos en el nivel dialógico. Del mismo modo, y en la dimensión social, la conversación progresa linealmente a partir de turnos y alternancias de turno. Y, en fin, el orden informativo se articula a partir de los que hemos denominado subactos.
} 
amplia, y desde sus primeras contribuciones, Briz y grupo Valesco (2003a, 2003b), hasta las más recientes, Briz y y Pons (2010), Hidalgo (2011) o Cabedo (2011), la labor realizada ha sido coherente y valiosa. También lo es, aunque no fuera concebida especialmente para su aplicación a la oralidad, la línea emprendida hace años por Garrido y cuyas últimas aportaciones (Garrido 2011a, 2011b y 2012) han venido a reforzar criterios previos. ${ }^{2}$ Asimismo, Cortés y Camacho (2005) o Fuentes (en prensa) se han ocupado de esta cuestión. Por el contrario, apenas ha existido interés alguno por las unidades relacionadas con lo temático-textual.

Supongamos un diálogo entre dos amigos que hace años que no se ven; habrá una serie de intervenciones con múltiples respuestas a la aludida pregunta que en este caso, tras los saludos, girará en torno a la salud, al tiempo transcurrido desde su último encuentro, a los aspectos más relevantes de cada uno en la actualidad, etc.; después, se podrán abordar otros temas; cuando estos se hayan agotado, aparecerán las despedidas, deseos de prosperidad, promesas de futuros encuentros o saludos a las personas allegadas; serán los temas, subtemas, sub(sub)temas, etc. (las subsecuencias). Paralelamente, esos contenidos se podrán clasificar según inicien la conversación, la desarrollen o la cierren. Esto mismo es de aplicación a una lección magistral, a una carta, a una conferencia o a un debate; en realidad es de aplicación a cualquier texto. Por eso, dentro del plano secuencial establecemos la división entre subsecuencias y secuencias. En ellas se alojan, a su vez, las unidades del plano enunciativo, que son de procesamiento: los enunciados, cuyas entidades menores son los actos y subactos. Cómo se presentan dos de las secuencias (inicio y cierre) y la integración en estas de las unidades subsecuenciales y de procesamiento son los objetivos de este artículo. Tales segmentaciones no solo servirán para poder describir mejor los discursos, sino también para dar una mayor coherencia a posteriores estudios enunciativos (cortesía, conectores, series enumerativas o mecanismos e intenciones en general).

Para este acercamiento, nos vamos a valer de un corpus de los discursos iniciales del presidente del Gobierno y del líder de la oposición en los "Debates en torno al estado de la Nación” (en adelante, DEN) celebrados en el presente siglo. ${ }^{3}$

\footnotetext{
Garrido prescinde de la distinción entre unidades «del sistema, gramaticales» frente a unidades de «la comunicación realizada, emitida», «del discurso ya producido», en términos de Fuentes (2000: 75 y 92). En su lugar, se propone que más allá de la oración las unidades existentes son también parte de la gramática. En otros términos, en lugar de unidades como los actos de discurso (como en Briz 2007) o los enunciados (Fuentes 2000), lo que hay más allá de la oración es secuencias de oraciones, construidas mediante un conjunto finito de relaciones denominadas relaciones retóricas o relaciones de discurso (Garrido 2009: 227); las oraciones solo existen y se construyen como constituyentes del discurso (Garrido 2010: 16). (Garrido 2011: 977)
}

3 Esta aproximación al estudio de los “Debates” que ofrecemos aquí, en cuanto a su extensión, se basa en un corpus delimitado en dos aspectos. En primer lugar, vamos a partir de los DEN celebrados en el presente siglo, o sea desde 2001 -en 2000 no hubo- hasta el último, el efectuado en 2011; en total 9 debates, que abarcarán tres legislaturas completas (VII, VIII y IX). En la primera de ellas, el Gobierno estaba presidido por J.M. Aznar y era Rodríguez Zapatero el líder de la oposición; se celebraron los debates en 2001, 2002 y 2003; en las dos restantes, Rodríguez Zapatero y M. Rajoy se reparten estos papeles (2005, 2006 y 2007, 2009, 2010 Y 2011). En segundo lugar, en este trabajo, la limitación viene impuesta por el contenido: nos vamos a ceñir a los discursos iniciales de los presidentes (Aznar y Rodríguez Zapatero) y de los líderes de la oposición (Zapatero y Rajoy) Para ello, nos valdremos de las transliteraciones aparecidas en el Diario de sesiones, así como de los vídeos correspondientes. Por tanto, el corpus a partir del cual se ha llevado a cabo este acercamiento consta de nueve discursos presidenciales de, aproximadamente, unos sesenta minutos de duración media y otros tantos del líder de la Oposición, de unos cincuenta minutos de duración media. 


\section{LOS INICIOS}

Un buen inicio (exordio), dicen los manuales de Retórica, ha de intentar ganar el interés del público y ayudar a crear una buena imagen del hablante; en él, no se ha de pensar tanto en captar la benevolencia de los interlocutores cuanto su confianza; se ha de evitar la longitud en favor de la brevedad y explicitud; se ha de huir de lo confuso y banal en aras de la claridad. Son los primeros minutos de una intervención y de ahí su importancia. A priori, podemos ver que en los discursos políticos analizados, hay una marcada diferencia entre los cierres de estos mismos discursos, ya vistos en Cortés (2012b), y los inicios a los que ahora nos referimos, donde no hay tanto lugar para el ornato y la solemnidad. Si en aquellos es frecuente un empleo especial de las pausas, la entonación, la anáfora, la concessio, el poliptoton, la preterición o la serie enumerativa ${ }^{4}$ para la consecución de ambas características, tales mecanismos apenas se dan en la secuencia inicio.

Aunque no en todos los discursos analizados, sí podemos hablar, tanto entre los emitidos por los presidentes como por los líderes de la oposición, de una tendencia (tras el preceptivo agradecimiento al presidente del Parlamento por concederle la palabra) a la segmentación de tales inicios en cuatro temas: tema de urgencia, tema de justificación del discurso, tema de interés y tema de adelanto de las partes del desarrollo. El siguiente cuadro recoge estas partes en los discursos de los debates analizados (Cuadro 2).

Cuadro 2. Los inicios y su segmentación en temas en los discursos del presidente y del líder de la oposición

\begin{tabular}{|c|c|c|c|c|c|c|c|c|}
\hline & & & $\begin{array}{l}\text { PRESI- } \\
\text { DENTES }\end{array}$ & & & & OPOSICIÓN & \\
\hline AÑO & $\begin{array}{c}\text { TEMA } \\
\text { DE } \\
\text { URGEN- } \\
\text { CIA }\end{array}$ & $\begin{array}{l}\text { JUSTI- } \\
\text { FICA- } \\
\text { CIÓN }\end{array}$ & $\begin{array}{c}\text { TEMA } \\
\text { DE } \\
\text { INTERÉS }\end{array}$ & ADELANTO & $\begin{array}{c}\text { TEMA } \\
\text { DE } \\
\text { URGENCIA }\end{array}$ & $\begin{array}{l}\text { JUSTIFI- } \\
\text { CACIÓN }\end{array}$ & $\begin{array}{c}\text { TEMA } \\
\text { DE } \\
\text { INTERÉS }\end{array}$ & $\begin{array}{l}\text { ADELAN- } \\
\text { TO }\end{array}$ \\
\hline 2001 & & $\begin{array}{l}\text { Justifi- } \\
\text { cación }\end{array}$ & & Metas/Ejes & & & $\begin{array}{c}\text { Tipo de } \\
\text { oposición }\end{array}$ & $\mathrm{X}$ \\
\hline 2002 & Perejil & $\begin{array}{l}\text { Justifi- } \\
\text { cación }\end{array}$ & & Ejes & $\begin{array}{l}\text { Perejil y } \\
\text { Euskadi }\end{array}$ & Alusión & $\begin{array}{l}\text { Empeoramiento } \\
\text { de la situación }\end{array}$ & $\begin{array}{c}\text { Repaso a la } \\
\text { situación }\end{array}$ \\
\hline 2003 & & $\begin{array}{l}\text { Justifi- } \\
\text { cación }\end{array}$ & $\begin{array}{l}\text { Constitu- } \\
\text { ción }\end{array}$ & Objetivos & & Alusión & $\begin{array}{c}\text { Autoritario y } \\
\text { eficaz }\end{array}$ & $\mathrm{X}$ \\
\hline 2005 & & & $\begin{array}{c}\text { Alabanza } \\
\text { españoles/ } \\
\text { Terrorismo }\end{array}$ & $\begin{array}{c}\text { Balance/ } \\
\text { Objetivos }\end{array}$ & Ferrol & & Idílico & $\mathrm{X}$ \\
\hline 2006 & & Alusión & $\begin{array}{l}\text { Logros del } \\
\text { Gobierno }\end{array}$ & Metas & ETA & & $\begin{array}{c}\text { Ufano e } \\
\text { ineficiente }\end{array}$ & $\mathrm{X}$ \\
\hline 2007 & Yemen & & $\begin{array}{l}\text { Logros del } \\
\text { Gobierno }\end{array}$ & Balance & Yemen & & Lamentable & Y de eso ... \\
\hline 2009 & & & Economía & Partes & & & $\begin{array}{c}\text { Desastre } \\
\text { económico }\end{array}$ & $\begin{array}{c}\text { Cada cosa } \\
\ldots\end{array}$ \\
\hline
\end{tabular}

$4 \quad$ Para el concepto y funcionamiento de las serie enumerativa, véase Cortés, ed. (2008). 


\begin{tabular}{|c|c|c|c|c|c|c|c|c|}
\hline 2010 & & Economía & Puntos de & & & $\begin{array}{c}\text { Ideas } \\
\text { contradictorias } \\
\text { en economía }\end{array}$ & \\
\hline 2011 & Afganistán & & Economía & $\begin{array}{c}\text { Aspectos } \\
\text { de la crisis } \\
\text { económica }\end{array}$ & Afganistán & $\begin{array}{c}\text { Justificación } \\
\text { irónica }\end{array}$ & $\begin{array}{c}\text { Desastre } \\
\text { económico }\end{array}$ & $\begin{array}{c}\text { Comenta- } \\
\text { rios sobre } \\
\ldots\end{array}$ \\
\hline
\end{tabular}

\subsection{Tema de urgencia}

Aparece cuando algún acontecimiento reciente se convierte en importante noticia para el país (conflicto con Marruecos por la isla de Perejil, muerte de turistas en Yemen, de soldados en Afganistán, etc.). A modo de ejemplo, ${ }^{5}$ Zapatero, en 2011, inicia su discurso lamentando la muerte de dos soldados españoles en Afganistán. El tema, en este caso, consta de tres subtemas, en los cuales se integrarán las unidades de procesamiento (enunciados, actos y subactos) (Cuadro 3).

Cuadro 3. Zapatero [2011]. Presentación del tema de urgencia

UNIDAD TEMÁTICO-TEXTUAL: TEMA DE URGENCIA. MUERTE DE SOLDADOS ESPAÑOLES EN AFGANISTÁN

SUBTEMA 1. Señor presidente, señoras y señores diputados [subacto 1], quiero mostrar el profundo pesar del

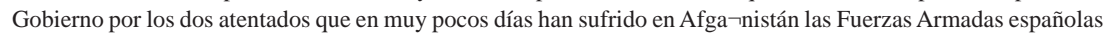
[subacto 2] [acto 1]. Como conse $\neg$ cuencia de ellos [subacto 3] han perdido la vida el sargento Manuel Argudin Perrino y la soldado Niyireth Pineda Marín [subacto 4] [acto 2]. También han resultado gravemente heridos el teniente Agustín Gras Báez y los soldados Jennifer García López, Abián Quevedo Santana, Ibrahim Maanan Ismael, Ruben Velázquez Herrera, Jhony Alirio Herrera Trejos y Roi Villa Souto, y el intérprete civil Menan Latifi Ashjari [acto 3]. [ENUNCIADO 1].

SUBTEMA 2. Que la mención de sus nombres sea muestra del home $\neg$ naje a su memoria, de la solidaridad con sus familias y del recuerdo a todos los que, miembros de los Ejércitos, de la Guardia Civil, de la Policía Nacional, de

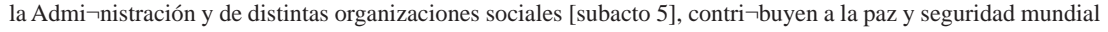
[subacto 6] [acto 4] y al auxilio de poblaciones maltratadas por la guerra, la violencia o el ansia de libertad [acto 5] [ENUNCIADO1].

SUBTEMA 3. Afganistán es hoy el más expresivo ejemplo de la necesidad de intervención de la comunidad internacional en escenarios convulsos[subacto 5] donde se ha per $\neg$ dido el valor de la vida [subacto 8] y donde se desconoce el sentido mismo de los derechos humanos [subacto 9] [acto 6]. Es también el que más esfuerzos exige [subacto 10], el que más sufrimiento causa [subacto 11], el más violento [subacto 12], el más desolador [subacto 13] [acto 7]. Pero también la comunidad internacional está conociendo experiencias esperanzadoras [acto 8], así ocurre en el Mediterráneo sur y también en el Medio Oriente [subacto 14], donde la libertad se abre caminos ines $\neg$ perados a los que España, como hizo desde su primera manifestación, prestará toda la ayuda que sus pueblos soliciten [subacto 15] [acto 9] [ENUNCIADO 2].

\begin{tabular}{|c|c|c|}
\hline TEMAS & SUB(SUB)TEMAS & ENUNCIADOS Y (SUB) ACTOS \\
\hline $\begin{array}{l}\text { TEMA1. Tema de } \\
\text { urgencia: } \\
\text { Afganistán }\end{array}$ & $\begin{array}{l}\text { Subtema 1: Dolor } \\
\text { Subtema2: Homenaje } \\
\text { Subtema3:Resumen actuación }\end{array}$ & $\begin{array}{l}\text { Enunciado } 1 \text { [actos } 1 \text { (subactos } 1,2 \text { ), } 2 \text { (subactos } 3 \text {, } \\
\text { 4) y 3] } \\
\text { Enunciado } 1 \text { [actos } 4 \text { (subactos 5, 6) y 5] } \\
\text { Enunciado } 2 \text { [actos } 6 \text { (subactos 7,8,9) 7.(subactos } \\
\text { 10,11,12,13) } 8 \text { y } 9 \text { (subactos } 14 \text { y 15)] }\end{array}$ \\
\hline
\end{tabular}

5 Al dedicar a cada uno de estos temas un artículo, que publicaremos próximamente, nos vamos a limitar en este capítulo introductorio a exponer un solo ejemplo de cada uno de ellos. 


\subsection{Justificación del discurso}

Habida cuenta de que se está en un acontecimiento muy señalado en el calendario político español, la justificación por parte de la persona que interviene en el acto no parece necesaria; no obstante, aunque fuera el tema menos empleado de los cuatro, se dio en algunas ocasiones [Aznar 2001, 2002 y 2003 o Zapatero 2002, 2003, 2006]. ${ }^{6}$ Un ejemplo es este de Aznar en 2002 (Cuadro 4).

Cuadro 4. [Aznar 2002] Unidades del discurso oral en el tema de justificación del discurso

TEMA DE JUSTIFICACIÓN. AZNAR Y SU PRESENCIA EN EL ACTO

SUBTEMA 1. Señoras y señores diputados [subacto 1], rendir cuentas ante el Parlamento es la esencia del régimen político que nos hemos dado [subacto 2] [acto 1] y ya es una costumbre asentada en nuestra democracia que el presidente del Gobierno solicite comparecer una vez al año ante la Cámara [acto 2], y a través de ella ante todos los ciudadanos [subacto 3], para dar cuenta de la labor realizada por el Ejecutivo [subacto 4] [acto 3] [ENUNCIADO 1].

SUBTEMA 2. Este es un buen momento para reivindicar la legitimidad de las Cáma $\neg$ ras como únicas depositarias de la soberanía nacional [acto 4]. Quienes formamos parte de ellas en virtud de unas elecciones generales somos los que damos expresión a la voluntad de los españoles [acto 5] [ENUNCIADO 2].

SUBTEMA 3. A mitad de la legislatura es momento de examinar los logros alcanzados entre todos [subacto 5] y señalar los objetivos para seguir avanzando [subacto 6] [acto 6] ENUNCIADO 3]. [Aznar 2002]

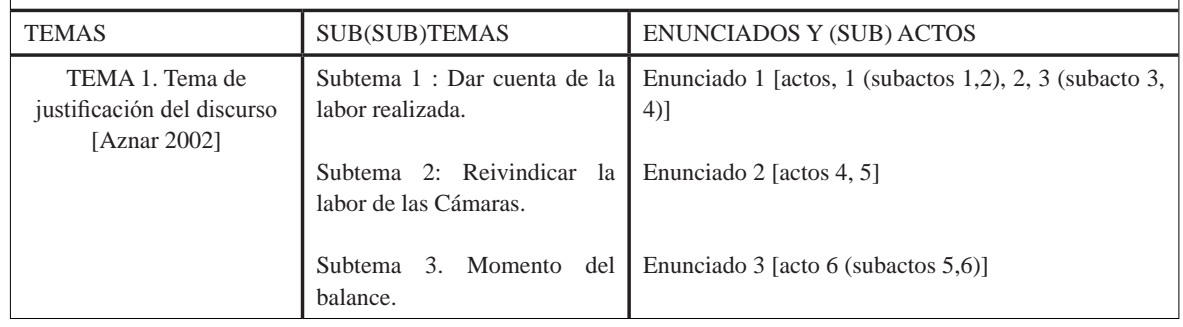

Zapatero se limita a su alusión en 2002, 2003 y 2006:

Señor presidente, señoras y señores diputados, iniciamos hoy el debate sobre política general que conocemos como el del estado de la Nación. Es, pues, un debate sobre la situación de España. Por ello, de lo que les quiero hablar hoy es de la España real, de lo que es España ahora y de lo que podemos hacer que España sea en el futuro [Zapatero 2006].

Y Rajoy solo recurre a este apartado en 2011 y lo hace como justificación para su ironía:

Señor presidente, señorías, el debate sobre el estado de la Nación surgió para enriquecer la vida parlamentaria con un análisis general de la situación española, una visión panorámica que el día a día no permite. Ahora, sin embargo, vivimos en la paradoja

6 Se señala en Vilches y otros (2007: 87) cómo el exordio (nuestro inicio) en su condición de toma de contacto entre el político y el auditorio es fundamental como introducción discursiva, por lo que es normal que este declare de manera expresa la razón por la que va a pronunciar su discurso. Sería un signo de prepotencia el no expresar la mencionada razón "pues quien pronuncia un discurso retórico está sometiéndose a la decisión de las personas a las que se dirige" (87). Por ello es normal que empiece tanto por la razón como por el tema. 
de que el debate extraordinario se nos ha convertido en cotidiano. Ya no es preciso reservar un día para este propósito. El estado de la nación ha alcanzado tal gravedad que cada vez que tomamos la palabra en esta Cámara no hablamos de otra cosa [Rajoy 2011].

\subsection{Tema de interés}

Se asocia con la aparición de algo en que se quiere hacer hincapié, por motivos diversos, bien por parte del Gobierno [en 2003, 2005, 2007, 2009, 2010 y 2011] bien por la oposición [en todos los debates]. En el primer caso, el tema es vario y puede ir de la importancia de la Constitución [Aznar 2003] a la economía [Zapatero 2009, 2010, 2011] pasando por los logros del Gobierno [Zapatero 2007]. En el segundo, la oposición suele utilizarlo (salvo en Zapatero 2001) para atacar lo que considera la mala gestión del Gobierno; generalmente, para enfatizar bien la ineficacia de su cometido [Zapatero 2002, 2003] o criticar la visión idílica ofrecida por el presidente en la sesión matinal (previa a este discurso) [Rajoy 2005, 2006 y 2007]. Veamos un ejemplo de este último hecho: el tema de interés de Rajoy [2005], cuya segmentación acometeremos según la disposición interna (segmentación en tres partes) propia de muchos temas ${ }^{7} \mathrm{y}$ algunos subtemas: presentación, razones que la justifican y conclusiones o consecuencias que se derivan de ambas:

Cuadro 5. Tema de interés en Rajoy [2005]: mala gestión del Gobierno

\begin{tabular}{|c|c|c|}
\hline \multicolumn{3}{|c|}{ TEMA 1. DISPOSICIÓN INTERNA. MALA GESTIÓN DEL GOBIERNO RAJOY [2005] } \\
\hline SUBTEMA 1 & SUBTEMA 2 & SUBTEMA 3 \\
\hline PRESENTACIÓN & RAZONES & CONCLUSIONES O CONSECUENCIAS \\
\hline $\begin{array}{l}\text { Señor presidente, } \\
\text { señorías, tras el idílico } \\
\text { panorama que nos ha } \\
\text { pintado el presidente del } \\
\text { Gobierno esta mañana, } \\
\text { la realidad es que si un } \\
\text { viajero hubiera estado } \\
\text { fuera de España durante } \\
\text { un año y regresara hoy } \\
\text { no daría crédito a lo } \\
\text { que ve. }\end{array}$ & 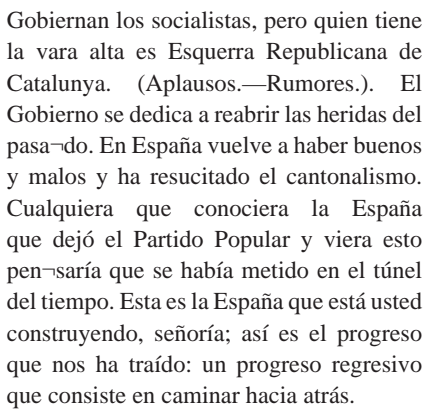 & $\begin{array}{l}\text { Esto es lo más lla } \neg \text { mativo en su primer año } \\
\text { de Gobierno, señor Rodríguez Zapatero: } \\
\text { su resuelta voluntad de avanzar con paso } \\
\text { firme hacia el pasado, como si la historia } \\
\text { hubiera esta } \neg \text { do esperando su providencial } \\
\text { advenimiento para dete } \neg \text { nerse, hacer tabla } \\
\text { rasa del presente, regresar a las caver } \neg \text { nas del } \\
\text { pasado para reconstruir la historia y volver a } \\
\text { comenzar. (Aplausos.-Rumores.) }\end{array}$ \\
\hline
\end{tabular}

\subsection{Tema de adelanto del desarrollo}

La última parte del inicio se suele dedicar, especialmente por parte del presidente, a anunciar los puntos que van a ser tratados en el desarrollo del discurso. Si hay diferencias entre los discursos del presidente y del líder de la oposición, este apartado las representan de forma muy clara: dicho adelanto siempre aparece cuando el que

\footnotetext{
Recordemos, por ejemplo, como Toulmin (1958) opinaba algo parecido para el párrafo, el cual se conformaría a partir de tres constituyentes: afirmación, que incluye la idea principal; información, donde se incorporan los datos que la apoyan, y garantía, en la que se evalúa la importancia de la información.
} 
habla es el presidente, por tanto es, en cuanto a su posible aparición, un tema fijo, y, además, es detallado, ya que se exponen los distintos apartados que se van a desarrollar. Así, se hablará en 2001, 2005 y 2007 del balance (sobre los aspectos en que se ha basado la política del Gobierno); en 2002, se habla de los ejes; en 2003, de los objetivos; de las metas, en 2006, etc. Veamos dos ejemplos [Zapatero 2006, 2009]:

un gran país en un momento de grandes oportunidades que los ciudadanos no quieren perder, que los ciudadanos no van a perder. Para ello, el Gobierno tiene como METAS, PRIMERO, más bienestar para todos los españoles y más oportunidades para los jóvenes; $S E G U N D O$, la extensión de los derechos civiles y sociales para los españoles y españolas, comenzando por los más necesitados;

TERCERO, una convivencia ordenada y segura de todos los españoles en una España plural y unida y CUARTO, la persecución de un orden internacional basado en la paz y en la cooperación [Zapatero 2006].

Cuadro 6. Tema de adelanto en Zapatero [2006]

\begin{tabular}{|c|l|l|}
\hline \multicolumn{2}{|c|}{ MECANISMOS DE ADELANTO DEL DESARROLLO EN EL INICIO. ZAPATERO [2006] } \\
\hline $\begin{array}{c}\text { Puntos que ha } \\
\text { de tratar en el } \\
\text { desarrollo }\end{array}$ & $\begin{array}{l}\text { Puntos que ha de tratar } \\
\text { en el desarrollo }\end{array}$ & $\begin{array}{l}\text { Primero más bienestar para todos los españoles y más oportunidades } \\
\text { para los jóvenes; } \\
\text { Segundo, la extensión de los derechos civiles y sociales para los } \\
\text { españoles y españolas, comenzando por los más necesi-tados; } \\
\end{array}$ \\
& $\begin{array}{l}\text { tercero, una convivencia ordenada y segura de todos los españoles } \\
\text { en una España plural y unida; } \\
\text { y cuarto, la persecución de un orden internacional basado en la paz } \\
\text { y en la cooperación. }\end{array}$ \\
\hline
\end{tabular}

En ocasiones, las partes, que ahora sustituyen a las metas aparecen segmentadas por consideraciones del propio presidente, lo que hace que la enumeración de las partes del desarrollo no aparezcan expresadas de manera tan clara; es más, aparecerá segmentada por la mayor o menor disquisición, lo que dificulta enormemente el poder conocer cuál será el orden de ideas en el desarrollo, precisamente la función más importante de este tema del inicio. Por ejemplo, es lo que ocurre en Zapatero [2009]:

Cuadro 7. Tema de adelanto en Zapatero [2009]

\begin{tabular}{|c|c|l|}
\hline \multicolumn{2}{|c|}{ MECANISMOS DE ADELANTO DEL DESARROLLO EN EL INICIO. ZAPATERO [2009] } \\
\hline $\begin{array}{c}\text { Puntos que ha } \\
\text { de tratar en el } \\
\text { desarrollo }\end{array}$ & $\begin{array}{c}\text { Las PARTES de su } \\
\text { intervención }\end{array}$ & $\begin{array}{l}\text { La primera parte de mi intervención estará dedicada a hacer un } \\
\text { breve balance de las medidas adop } \text {-tadas y a anunciar nuevas } \\
\text { iniciativas concretas para proponer a la Cámara frente a los desafíos } \\
\text { que España debe afrontar, propuestas para el corto y el largo plazo } \\
\text { En la segunda parte me referiré a compromisos de orden distinto al } \\
\text { económico-social que el Gobierno quiere asumir para el próximo } \\
\text { periodo de sesiones, }\end{array}$ \\
\hline
\end{tabular}

Posteriormente, tras aclara este último punto en cuanto a su desarrollo, el presidente Zapatero hace una larga consideración introductoria adicional:

pero antes permítanme una consideración introductoria adicional. Las decisiones que adoptemos en estos meses sobre nuestro futuro modelo de crecimiento van a ser 
determinantes para el futuro de nuestro país. Estoy plenamente convencido de ello. No saldremos de la crisis sin cambios, sin hacer determinadas opciones y sin ser plenamente consecuentes con ellas. El Gobierno que presido va a ser fiel a los valores que defiende y a los compromisos contraídos con sus electores. No podía ser de otra forma y no va a ser de otra forma. Pero también soy consciente de que en esta etapa difícil y decisiva es necesario conjugar esa fidelidad con la voluntad de propiciar la conciliación de ideas propuestas y sensibilidades diversas. Lo voy a hacer, lo voy a intentar hasta donde sea posible. Lo haré en tres ámbitos diversos, pero compatibles: en el del diálogo social, en el de la relación entre administraciones y, por supuesto, aquí, en el Parlamento.

Y termina el apartado con esta consideración del adelanto:

En relación con los tres, formularé también hoy haré propuestas concretas a la Cámara.

\section{LOS CIERRES}

En otro artículo (Cortés 2012b), analizamos el papel de las series enumerativas en los cierres presidenciales y observamos que todos ellos, sean emitidos por presidentes de una u otra ideología, respondían a un mismo esquema. Comienzan con un vocativo; a continuación, se trata el tema de los ideales de partida; más tarde, se pasa al tema de las realidades llevadas a cabo por el Gobierno; se continúa con el tema de las cuestiones que quedan pendientes para el futuro e inmediatamente se suele dejar unos segundos para el tema del halago, a través de la potenciación de los valores de España o de los españoles (libertad, dignidad, etc.); se termina con el agradecimiento por la atención. Si exceptuamos los ideales, de los que se prescinden en cuatro ocasiones -tres de ellas coincidiendo con los años de cierre del mandato- y el halago de los españoles, omitido en 2003, 2009 y 2011 y sustituido por otros temas, el resto de los apartados citados se dan en todos los cierres presidenciales, ${ }^{8}$ tal y como se puede ver en el cuadro siguiente:

Cuadro 8. Secuencias temáticas del cierre en los discursos presidenciales ${ }^{9}$

\begin{tabular}{|c|c|c|c|c|c|c|c|c|c|}
\hline PARTES & 2001 & 2002 & 2003 & 2005 & 2006 & 2007 & 2009 & 2010 & 2011 \\
\hline Vocativos & $\mathrm{S} . \mathrm{P}$. & S.P/S. & $\mathrm{S} . \mathrm{P} / \mathrm{S}$. & $\mathrm{S}$. & $\mathrm{S}$. & $\mathrm{S} . \mathrm{P} / \mathrm{S}$. & $\mathrm{S}$. & $\begin{array}{c}\text { Sr/Sra } \\
\text { Diput }\end{array}$ & $\mathrm{S}$. \\
\hline IDEALES & $\mathrm{X}$ & $\mathrm{X}$ & & $\mathrm{X}$ & & & $\mathrm{X}$ & $\mathrm{X}$ & \\
\hline REALIDAD & $\mathrm{X}$ & $\mathrm{X}$ & $\mathrm{X}$ & $\mathrm{X}$ & $\mathrm{X}$ & $\mathrm{X}$ & $\mathrm{X}$ & $\mathrm{X}$ & $\mathrm{X}$ \\
\hline FUTURO & $\mathrm{X}$ & $\mathrm{X}$ & $\mathrm{X}$ & $\mathrm{X}$ & $\mathrm{X}$ & $\mathrm{X}$ & $\mathrm{X}$ & $\mathrm{X}$ & $\mathrm{X}$ \\
\hline HALAGOS & $\mathrm{X}$ & $\mathrm{X}$ & $\mathrm{X}$ & $\mathrm{X}$ & & $\mathrm{X}$ & & $\mathrm{X}$ & \\
\hline OTROS & & & & & Terrorismo & & Comp. \\
social & & Respeto ${ }^{1}$ \\
\hline Agradec. & $\begin{array}{c}\text { M.G. } \\
\text { S.P. }\end{array}$ & $\begin{array}{c}\text { M.G. } \\
\text { S.P. }\end{array}$ & M.G. & M.G. & M.G. & M.G. & M.G. & M.G. & G. \\
\hline
\end{tabular}

Hasta ahora solo hemos estudiado los cierres emitidos por los presidentes y no por los líderes de la oposición.

$9 \quad$ S.P $=$ Sr/a Presidente/a. S = Señorías. Sr/Sra Diput $=$ Señoras y señores diputados. M.G. $=$ Muchas gracias. G. Gracias. 
En este apartado, intentamos ver, como hemos hecho en los inicios, la estructura secuencial de estos cierres: su segmentación en temas y la integración en ellos de los subtemas, sub(sub)temas, así como de las unidades de procesamiento, que se integran en estas.

\subsection{Tema de ideales}

Las ilusiones, los objetivos, las intenciones o los propósitos que guían a un presidente al llegar al poder son mencionados en este primer tema del cierre, tras los vocativos correspondientes (señorías, señoras y señores diputados, etc.). Así, en Zapatero [2010] tal tema lo expresa de esta manera:

Cuadro 9. Tema de ideales en el cierre del discurso [Zapatero 2010].

\begin{tabular}{|c|c|c|}
\hline \multicolumn{3}{|c|}{ CIERRE Y TEMA DE IDEALES } \\
\hline \multicolumn{3}{|c|}{$\begin{array}{l}\text { SUBTEMA 1. El esfuerzo colectivo que requiere la aplicación de las reformas económicas es necesario [acto } \\
\text { 1], tiene pleno sentido [acto 2]. Es necesario para que volvamos a crecer [subacto 1] y lo hagamos con fuerza y } \\
\text { sostenibi-lidad [subacto 2] [acto 3]; es necesario para que volvamos a crear empleo [acto 4]; es necesario para } \\
\text { preservar el Estado de bienestar [subacto 3], asen-tado en esas cuatro firmes columnas que son la educa`ción, } \\
\text { la sanidad pública, las pensiones [subacto 4] y ahora también el sistema de dependencia [subacto 5] [acto 5]; es } \\
\text { necesario para mantener la política de incremento de becas que hemos hecho [acto 6], para asegurar la renta de } \\
\text { emancipación a los jóvenes que hemos puesto en marcha [subacto 6] y hacer efectivo el derecho a una vivienda } \\
\text { en condiciones asequibles [subacto 7] [acto 7]; es necesario para defender la conciliación de la vida familiar y } \\
\text { laboral [subacto 8], aplicando y extendiendo el permiso de paternidad que hemos puesto en marcha [subacto } \\
\text { 9] [acto 8]; es necesario para proseguir la mejora de las pensiones mínimas [subacto 10] como hemos hecho } \\
\text { en estos últimos seis años [subacto 11] [acto 9], y es necesario, en fin, para man-tener la cohesión social en } \\
\text { nuestro país [subacto 12], que es la máxima aspiración del grupo político al que represento [subacto 13] [acto } \\
\text { 10].[ENUNCIADO 1] }\end{array}$} \\
\hline TEMAS & SUB(SUB)TEMAS & ENUNCIADOS Y (SUB) ACTOS \\
\hline $\begin{array}{l}\text { Tema de ideales en el } \\
\text { cierre de Zapatero [2010] }\end{array}$ & $\begin{array}{l}\text { Subtema } 1: \text { Es esfuerzo } \\
\text { colectivo y su necesidad }\end{array}$ & $\begin{array}{l}\text { ENUNCIADO } 1 \text { [actos, } 1 \text { 2, } 3 \text { (subactos } 1,2) 4,5 \\
\text { (subactos, 3, 4, 5) 6, } 7 \text { (subactos 6, 7) } 8(\text { subactos } 8,9) \\
9 \text { (subactos } 10,11) 10 \text { (subactos } 12,13) \text { ] }\end{array}$ \\
\hline
\end{tabular}

\subsection{Tema de realidades}

El presidente en este tema va a defender cómo se ha pasado de los ideales a su aplicación en el presente, lo que supone siempre un éxito de su política y, por tanto, digno de aparecer en este momento esencial del discurso.

Ya vimos que el discurso en sí forma un continuum en el que, a su vez, es frecuente que los temas $\mathrm{y}$, en menor medida, los subtemas, además de incorporarse en unidades mayores (secuencias) e integrar a otras menores (enunciados, actos, subactos), tengan su disposición interna que venga a coincidir con subsecuencias, en el primer caso, y sub(sub)secuencias, en el segundo; tal disposición consta, tal y como ya señalamos al presentar el Cuadro 7, de tres partes: a) presentación del tema; b) razones que sustentan tal opinión; y c) constatación del hecho y consecuencias que se puedan derivar.

Veamos todo lo dicho en el apartado que Zapatero [2009] dedica en su cierre al tema realidad (Cuadro 10). 
Cuadro 10. Presentación del tema de la realidad en el cierre. Zapatero [2009]

\begin{tabular}{|c|c|c|}
\hline \multicolumn{3}{|c|}{ CIERRE: TEMA DE URGENCIA } \\
\hline \multicolumn{3}{|c|}{$\begin{array}{l}\text { PRESENTACIÓN DEL TEMA } \\
\text { [SUBTEMA 1.]. Ese compromiso se ha ido haciendo realidad [acto 1] }\end{array}$} \\
\hline \multicolumn{3}{|c|}{ RAZONES QUE SOSTIENEN TAL OPINIÓN } \\
\hline \multicolumn{3}{|c|}{$\begin{array}{l}\text { SUBTEMA 2.]. Es un salario mínimo que ha crecido el } 35 \text { por ciento en estos años para los traba-jadores que } \\
\text { menos ganan en nuestro país [acto 2]; son las pen-siones mínimas, que han crecido al } 31 \text { por ciento desde } 2004 \\
\text { [subacto 1], beneficiando a } 3 \text { millones de pensionistas con las rentas más bajas [subacto 2] [acto 3] (Aplausos.); } \\
\text { son las más de } 650.000 \text { personas en situación de dependencia que por primera vez han visto reconocida su } \\
\text { situación [subacto 3] y perciben prestaciones y ayudas de la sociedad [subacto 4] [acto 4]; son los más de } \\
1.500 .000 \text { jóvenes que disfrutan hoy de una beca [subacto 5], } 300.000 \text { más que en } 2004 \text { [subacto 6] [acto5]; son } \\
\text { los } 100.000 \text { jóvenes que perciben la renta de emancipación para la ayuda a vivienda en alquiler [acto 6]; son los } \\
350.000 \text { varones que han disfrutado del permiso de paternidad desde } 2007 \text { [subacto 7] o las } 800.000 \text { familias que } \\
\text { han percibido } 2.500 \text { euros por el nacimiento de cada uno de sus hijos [subacto 8] [acto 7]. }\end{array}$} \\
\hline \multicolumn{3}{|c|}{ CONSTATACIÓN DEL HECHO Y CONSECUENCIAS } \\
\hline \multicolumn{3}{|c|}{$\begin{array}{l}\text { SUBTEMA 3. Son pues millones de jóvenes, de personas mayores, de discapacitados, de dependientes [acto 8], } \\
\text { son millones de familias, las más necesi-tadas socialmente [subacto 9], que han obtenido el apoyo del con-junto } \\
\text { de los ciudadanos a través de las propuestas del Gobierno [subacto 10] [acto 9] [ENUNCIADO 1]. (Aplausos.) }\end{array}$} \\
\hline TEMA & SUBTEMA & ENUNCIADOS Y (SUB) ACTOS \\
\hline $\begin{array}{l}\text { TEMA1. Realidades } \\
\text { sociales }\end{array}$ & $\begin{array}{l}\text { Subtema 1. Presentación } \\
\text { Subtema 2. Razones } \\
\text { Subtema 3. Consecuencias }\end{array}$ & $\begin{array}{l}\text { ENUNCIADO 1: [actos 1,2,3 (subactos } 1,2), 4 \\
\text { (subactos } 3,4), 5 \text { (subactos } 5,6), 7 \text { (subactos } 7,8), 8 \text { y } \\
9 \text { (subactos } 9 \text { y } 10 \text { ) }\end{array}$ \\
\hline
\end{tabular}

\subsection{Tema del futuro}

Apartado ineludible en los cierres es que el presidente hable de sus proyectos, del futuro, de lo que queda por hacer, pues es de vital importancia el que los ciudadanos sean conscientes de que se parte de un ideal, se consigue un presente mucho mejor (tema de la realidad) gracias al esfuerzo de todos, especialmente al buen hacer del Gobierno, pero nada de ello es impedimento para que el presidente sepa que quedan cosas por hacer (tema futuro); con ello, se intentará mantener la esperanza de los ciudadanos con lo que aún han de conseguir (Cuadro 11).

Cuadro 11. Presentación del tema del futuro en el cierre Zapatero [2010].

\begin{tabular}{|c|}
\hline CIERRE: TEMA DEL FUTURO \\
\hline DISPOSICIÓN INTERNA Y MECANISMOS CONEXIÓN \\
\hline PRESENTACIÓN DEL TEMA \\
SUBTEMA 1.-. No va a ser una tarea fácil [subacto 1], pero hemos de acometerla [subacto 2] y sabremos hacerlo \\
(subacto 3) [acto 1] [ENUNCIADO 1]. \\
\hline RAZONES QUE SOSTIENEN TAL OPINIÓN \\
$\begin{array}{l}\text { SUBTEMA 2.- El momento es decisivo para engan`charnos definitivamente al grupo de países más compe } \neg \text { titivos } \\
\text { de Europa [subacto 4], al que siempre hemos querido perte-necer [subacto 5] [acto 2] [ENUNCIADO 2]. }\end{array}$ \\
\hline
\end{tabular}


SUBTEMA 3.- Podemos negarnos a acometer esta modernización [subacto 6] y correr el riesgo de languidecer [subacto 7], de quedarnos descolᄀgados [subacto 8] [acto 3], o podemos afrontar la necesidad de los cambios [subacto 9] para dirigirlos en la mejor dirección [subacto 10] [acto 4], hacer de la nece $\neg$ sidad virtud [subacto 11] y crear nuestro propio destino [subacto 12] [acto 5], porque este está en nuestras manos [subacto 13] aunque nos cueste esfuerzos [subacto 14 [acto 6], y nos va a costar esfuerzos [acto 7] [ENUNCIADO 3]

\begin{tabular}{|c|c|c|}
\hline TEMA & SUBTEMA & ENUNCIADOS Y (SUB) ACTOS \\
\hline \multirow{3}{*}{$\begin{array}{c}\text { TEMA. Tareas que quedan } \\
\text { para el futuro [Zapatero } \\
\text { 2010]] }\end{array}$} & $\begin{array}{l}\text { Subtema } 1 \text { : Presentación de } \\
\text { la tarea }\end{array}$ & Enunciado 1 [actos, 1 (subactos 1,2, 3)] \\
\hline & $\begin{array}{l}\text { Subtema 2:Causas que la } \\
\text { hacen necesaria. }\end{array}$ & Enunciado 2 [acto 2 ( subactos 4,5 )] \\
\hline & $\begin{array}{l}\text { Subtema 3. Mitad de la } \\
\text { legislatura. }\end{array}$ & $\begin{array}{l}\text { Enunciado } 3 \text { [acto3 (subactos } 6,7,8) 4 \text { (subactos } 9,10) \\
5 \text { (subactos } 11,12) 6 \text { (subactos } 13,14) 7 \text { ] }\end{array}$ \\
\hline
\end{tabular}

\subsection{Tema del halago}

En estos debates analizados, salvo en 2006, 2009 y 2011, en que fue sustituido por temas de máximo interés, nuestros presidentes dedican un tiempo a halagar a los españoles o a España como país. Por ejemplo, Aznar, en 2003, señala su confianza en todos los españoles, en sus energías y en sus esfuerzos para hacer un país mejor: "Yo creo en ese futuro y pienso que todos los españoles, sean cuales sean nuestras ideas y nuestras aspiraciones, podemos sumar energías y esfuerzos para hacer de España un país cada día mejor”. Zapatero, en 2005, ha comprobado muchas veces en su primer año de mandato lo inteligentes, lo sensatos y demás que somos los españoles: "cuánta sensatez, cuánta generosidad, cuánto talento albergan nuestras gentes”. Son todas frases con poco contenido y tendentes a persuadir en su vanidad al futuro votante. Zapatero, en 2007, termina su intervención manifestando su plena confianza en todos nosotros, sin excepciones: "Tengo una confianza plena en la sociedad española, en su capacidad, en su tesón, en sus valores, en su futuro". El propio líder socialista, tres años después, en 2010, va a sentir la complacencia propia del orgullo de quien tanto ha tenido que ver en el cambio positivo de España, que ahora es otro país:

En este tiempo España se ha convertido en otro país, en un país del que podemos sentirnos orgullosos, en un país tolerante, abierto, moderno, en el que se puede vivir a gusto. Hemos sido tan capaces que la confianza en nosotros mismos no es una cuestión de fe, es una cuestión de memoria y de país [Zapatero, 2010].

Ninguna, posiblemente, más determinante que la de Aznar [2002]:

Los españoles confiamos en nosotros mismos y eso es lo que nos permite plantearnos nuevos retos para nuestro futuro. Somos una sociedad más libre, más fuerte, más desarrollada y más decidida a seguir avanzando, y estos creo que son los verdaderos rasgos distintivos del estado de nuestra nación [Aznar, 2002].

\subsection{0 tros temas}

Los tres años en que Zapatero prescinde del halago lo sustituye por temas de máximo interés. En 2011, consciente de que su presidencia acababa y de que era su 
último debate, el líder socialista aprovecha este momento para subrayar su talante como presidente: un presidente, por encima de todo, respetuoso; de hecho, la palabra respeto aparece repetida en seis ocasiones y ubicada en el inicio de varios actos, lo que es muy indicativo, tal y como veremos en el artículo dedicado a 0 tros temas. El texto es este:

De ahí que mi actitud siempre que he subido a hacer un debate sobre el estado de la Nación haya sido de respeto. De respeto, en primer lugar, a los ciudadanos, a quienes nos debemos; de respeto a esta institución, a la Cámara que encarna la soberanía popular; de respeto a todos los grupos y a sus señorías. Ese respeto es aún más profundo, para mí, a mi país, a España, sobre la que expreso mi más absoluta confianza en su futuro, y deseo expresar mi agradecimiento a todos los grupos políticos que, con crítica o reproches, vienen - y lo han hecho en unos momentos más y en otros menos—colaborando con el Gobierno. Mi respeto a todos los grupos y mi gratitud a aquellos que han colaborado. Y al Grupo Socialista, mi más profunda gratitud por su lealtad, por su compromiso y por su responsabilidad, el sentido de la responsabilidad que se espera de todos nosotros hoy, mañana y todos los días del futuro [Zapatero 2011].

Ante los problemas económicos que comienzan a acuciar a los españoles, en 2009, Zapatero sustituye el halago por el compromiso social con los más débiles, con los primeros a quienes ha empezado a afectar la crisis.

Quiero hoy reiterar, en nombre del Gobierno y del proyecto que represento, que este es nuestro principal compromiso y que, por tanto, mantendremos y fortaleceremos todas las políticas sociales que hemos ido conquistando en los últimos cinco años. Es mi mayor compromiso y también mi principal deber con los ciudadanos que nos dieron su apoyo electoral. Hoy extiendo ese compromiso de manera singular a los que en este momento más necesitan de la solidaridad y del apoyo de todos, las personas sin empleo. Ellas son destinatarias, con la misma fuerza y el mismo fundamento, de nuestro compromiso social acreditado, el que ha guiado toda nuestra acción política y la seguirá guiando en el futuro, para siempre [Zapatero 2011].

Finalmente, en 2006 el líder socialista opta por el tema del terrorismo. Era el momento en que Zapatero había apostado por acabar con él mediante un pacto con la banda terrorista ETA, pacto que, desgraciadamente, saltó por los aires meses después del debate, cuando esos mismos terroristas colocaron una bomba en Barajas (30 de diciembre de 2006) que costó la vida a dos ciudadanos de origen ecuatoriano. Pero en el momento del DEN era la gran apuesta de Zapatero, y de ahí su lugar destacado en el cierre de su discurso:

No quiero terminar, señorías, sin referirme al problema que más ha angustiado a la sociedad española desde la transición. Desde el recuerdo sentido y emocionado, y desde el apoyo a todas las víctimas del terrorismo quiero afirmar que nos hemos ganado como sociedad el derecho al fin de la violencia, a la paz y a la esperanza de convivir juntos tan solo sometidos a los mandatos de la libertad y de la ley. Reafirmo hoy mi determinación de trabajar para el fin de la violencia, para el fin de ETA. Confío en que pueda ser una tarea de todos porque todos lo merecemos, confío en que todos actuemos con generosidad porque a todos nos lo demandan los españoles. Por mi parte comprometo nuevamente mi disposición al máximo diálogo para recorrer el camino a la paz (Aplausos.) [Zapatero 2006]. 
Cuadro 12. Tema del terrorismo en “Otros temas” del cierre. Zapatero [2006]

\begin{tabular}{|c|l|l|}
\hline \multicolumn{2}{|c|}{ DISPOSICIÓN INTERNA. LA PRETENSIÓN DE UN PRESIDENTE: EL FIN DEL TERRORISMO } \\
[ZAPATERO 2006]
\end{tabular}

\section{Conclusiones}

El análisis de discursos amplios, como pueden ser las intervenciones iniciales del presidente del Gobierno y del líder de la oposición en los DEN, exige unidades de segmentación superiores a las de procesamiento (enunciados, actos, subactos). Esa necesidad nos llevó a plantearnos la aplicación de otras unidades, pertenecientes al plano secuencial. En el presente artículo hemos podido ver las aportaciones que a dichos análisis ofrece la unidad secuencial inicio y su división en subunidades temático-textuales (tema de urgencia, tema de justificación, tema de interés y tema de adelanto). Asimismo, podemos observar cómo se integran los elementos de los diferentes planos en un continuum. Es verdad que el desarrollo de tales cuestiones y su acercamiento implica un estudio mucho más detallado, tal y como hemos hecho en los artículos dedicados a cada uno de los cuatro temas del inicio y hemos de hacer en los del cierre, donde también hemos encontrado temas característicos (ideales, realidad, futuro, halagos y otros). Ahora, solo hemos esbozado y adelantado nuestro sistema de unidades, que va más allá del enunciado y que nos permite la segmentación y análisis de amplios discursos. Pero no es solo esto, sino que en los análisis enunciativos, estudio de los mecanismos mediante los cuales conseguimos efectos (cortesía, ironía, argumentación, racismo, et.), nos permitirá comparar fragmentos del discurso semejantes; pensamos que en tales acercamientos no siempre puede ser confrontables un tema de urgencia con cualquier otro apartado del discurso, por citar un ejemplo. 


\section{BiBILOGRAFÍA}

Briz, Antonio y Grupo Valesco. 2003a. "Un sistema de unidades para el estudio del lenguaje coloquial”. Oralia 6: 7-61.

Briz, Antonio y Grupo Valesco. 2003b. "Las unidades de la conversación: el acto” Estudios ofrecidos al profesor J osé J esús de Bustos Tovar. II. Madrid: Universidad Complutense. 953-968.

Briz, Antonio y Salvador Pons. 2010. "Unidades, marcadores discursivos y posición” LoS estudios sobre marcadores del discurso, hoy. O.Loureda y Esperanza Acín, Eds. Madrid: Arco/Libros. 327-358.

Cabedo, Adrián. 2011. "Hacia un modelo predictivo para la segmentación prosódica del discurso oral coloquial: Mestel (Modelo Estadístico para la Selección de Términos Entonativos Ligados)". O ralia 14: 85-104.

Cortés, Luis. 2012a. "Los límites del discurso: condicionantes y realizaciones”. CLAC. Círculo de Lingüística A plicada a la Comunicación 51: 3-49.

Cortés, Luis. 2012b. "La serie enumerativa en el cierre de los discursos”. Estudios F ilológicos 49: 39-57.

Cortés, Luis. En prensa. "El tema de urgencia en los D ebates del E stado en torno a la Nación”. BFUCh, 48,2 (2013).

Cortés, L. Edit. 2008. La serie enumerativa en el discurso oral en español. Madrid: Arco/ Libros.

Cortés, L. y M. ${ }^{a}$ Matilde Camacho. 2005. Unidades de segmentación y marcadores del discurso. Madrid: Arco/Libros.

Fuentes, Catalina. 2000. Lingüística Pragmática y Análisis del discurso. Madrid: Arco Libros.

Fuentes, Catalina (2013). "La gramática discursiva: niveles, unidades y planos de análisis”. Cuadernos AISPI 2: 15-36.

Garrido, Joaquín, 2009. M anual de lengua española. Madrid: Castalia.

Garrido, Joaquín, 2010. “Thème et dislocation dans la langue parlée: De la syntaxe de la phrase à la construction du discours”. C LAC. Círculo de L ingüística Aplicada a la Comunicación 42: 3-21.

Garrido, Joaquín, 2011a "Unidades y relaciones en la construcción del discurso”. Actas del IX Congreso de Lingüística G eneral. Valladolid: Universidad de Valladolid. 976-990.

Garrido, Joaquín. 2011b. "Las unidades del discurso”. 60 problemas de gramática dedicados a I gnacio Bosque. Eds. M. ${ }^{a}$ V. Escandell, M. Leonetti y C. Sánchez López. Madrid: Akal. 420-426.

Garrido, Joaquín, 2012. ”Narración y argumentación en la construcción de discurso”. Actas del XVI Congreso Internacional de la ALFAL. Alcalá de Henares: Universidad de Alcalá de Henares. 1095-1103. http://alfal2011.mundoalfal.org/\#/pdf/121alfal.pdf

Hidalgo, Antonio. 2011: "Segmentación y discurso oral: notas sobre el papel demarcativo de la prosodia en la conversación”. Sintaxis y análisis del discurso hablado en español. Homenaje a Antonio Narbona, I. Eds. J.J. de Bustos et alii. Sevilla: Universidad. 237258.

Toulmin, Stephen. 1958: The uses of Argument. Cambridge: Cambridgty Press.

Vilches, Fernando et al. 2007. M anual de Retórica parlamentaria de la Asamblea de Madrid. Madrid: Asamblea de Madrid. 